\title{
$A$ priori filtered chemical source term modeling for LES of high Karlovitz number premixed flames
}

\author{
Simon Lapointe ${ }^{a, 1}$ and Guillaume Blanquart ${ }^{b}$ \\ ${ }^{a}$ Graduate Aerospace Laboratories, California Institute of Technology, Pasadena, CA \\ 91125, USA \\ ${ }^{b}$ Mechanical Engineering Department, California Institute of Technology, Pasadena, CA \\ 91125, USA
}

\begin{abstract}
Assumptions behind closure models for the filtered source term are studied a priori using results from DNS of turbulent $n$-heptane/air premixed flames at varying Karlovitz numbers. Simulations with both detailed chemistry and tabulated chemistry, as well as unity and non-unity Lewis numbers, are used to determine if finite-rate chemistry and differential diffusion effects affect the filtered chemical source terms. While the unfiltered source term shows large fluctuations, the filtered source terms from detailed chemistry and tabulated chemistry are in good agreement at sufficiently large filter widths $\left(\Delta \gtrsim l_{F}\right)$. Using the concept of optimal estimators, it is shown that a tabulation approach using the filtered progress variable and its variance can predict accurately the filtered chemical source terms. Finally, the filtered source terms from the DNS are compared to predictions from two commonly assumed sub-filter probability density function models. Both models show deviations from the filtered DNS source terms but predict accurately the mean turbulent flame speed. The results illustrate the potential of using simple tabulated chemistry approaches based on presumed PDFs for LES of premixed flames in the thin and distributed reaction zones regimes.
\end{abstract}




\section{Introduction}

While Direct Numerical Simulations (DNS) are an extremely valuable research tool, they are currently limited to canonical configurations and low Reynolds numbers. This is due to the extreme range of length and time scales separating the large, energy-containing eddies, and the small, energy-dissipating eddies. At high Reynolds numbers, the resolution of all the scales of a turbulent flow requires computing resources well beyond current capabilities.

As an alternative, Large Eddy Simulations (LES) resolve the larger eddies while the effects of the smaller eddies are modeled. Depending on where the resolution threshold is set, the range of resolved scales can be drastically reduced, making LES of practical geometries and at relevant conditions computationally feasible. The reduction in the range of resolved scales is often accompanied by the use of reduced-order chemistry models [1]. This helps reduce computational cost and simplify the sub-filter closure of non-linear terms.

Among such chemistry reduction methods, chemistry tabulation $[2,3]$ is an appealing approach as it requires the transport of only a few controlling variables (usually one or two). All thermodynamic and flame properties are tabulated against these controlling variables using information obtained from detailed chemistry simulations of laminar flames. Commonly used tabulated chemistry modeling approaches

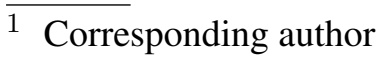
Email address: slapointecal tech. edu (S. Lapointe) 
for LES include the G-equation [4], PCM-FPI [5], and physical-space filtered flamelets [612]. While these models have been used with success in LES, only a very limited number of studies have targeted premixed flames at high Karlovitz numbers [13, 14].

Most tabulated chemistry approaches rely on the assumption that the turbulent flame behaves locally like a one-dimensional laminar flame (flamelet regime). At high Karlovitz numbers, small turbulent eddies broaden the preheat zone and disturb the reaction zone $[15,16]$, causing significant fluctuations in the chemical source terms [17] and local extinctions due to differential diffusion in high curvature regions [18]. Tabulated chemistry simulations using a single progress variable do not capture these phenomena [19]. Therefore, it is unclear if current sub-filter closure models, validated using tabulated chemistry results $[8,10]$, are still valid in the thin and distributed reaction zones regimes.

In traditional chemistry tabulation for low Karlovitz number turbulent flames, the filtered chemical source term of the progress variable, $\tilde{\dot{\omega}}_{c}$ (where ${ }^{\sim}$ denotes densityweighted spatial filtering), is expressed as a function of the filtered progress variable, $\tilde{c}$, and its variance, $c_{v}=\widetilde{c c}-\tilde{c} \tilde{c}$ (or, equivalently, the unmixedness $S_{c}=$ $\left.c_{v} /\left[\tilde{c}\left(c_{b}-\tilde{c}\right)\right]\right)$. More precisely, the dependence of $\tilde{\dot{\omega}}_{c}$ on $\tilde{c}$ and $c_{v}$ is obtained through the sub-filter probability density function (PDF) of the progress variable:

$$
\tilde{\dot{\omega}}_{c}\left(\tilde{c}, c_{v}\right)=\int_{0}^{c_{b}} \dot{\omega}_{c}(c) P\left(c \mid \tilde{c}, c_{v}\right) \mathrm{d} c
$$

where $\dot{\omega}_{c}$ is the unfiltered progress variable source term, $P\left(c \mid \tilde{c}, c_{v}\right)$ is the sub-filter PDF of $c$ at a given $\tilde{c}$ and $c_{v}$, and the progress variable is normalized between 0 and $c_{b}$. Two assumptions are involved in Eq. (1): 
(1) None of the fluctuations of $\dot{\omega}_{c}$ at a given $c$ are necessary $\left(\dot{\omega}_{c} \rightarrow \dot{\omega}_{c}(c)\right)$.

(2) Only two quantities are sufficient to express $\tilde{\dot{\omega}}_{c}$, namely $\tilde{c}$ and $c_{v}\left(\tilde{\dot{\omega}}_{c} \rightarrow\right.$ $\left.\tilde{\dot{\omega}}_{c}\left(\tilde{c}, c_{v}\right)\right)$.

Shall these two assumptions be valid, there are two unknowns functions:

(1) The sub-filter PDF, $P\left(c \mid \tilde{c}, c_{v}\right)$.

(2) The unfiltered chemical source term, $\dot{\omega}_{c}(c)$.

It is the goal of this paper to investigate these assumptions and assess common expressions for $P\left(c \mid \tilde{c}, c_{v}\right)$ and $\dot{\omega}_{c}(c)$. This will be done a priori using published DNS of turbulent premixed flames. These simulations were used in recent work to study the effects of turbulence on the chemical source terms [17] and to study the effects of the flame on vorticity characteristics [20]. The DNS dataset used is reviewed in Section 2, the methodology is described in Section 3, assumptions 1 and 2 are assessed in Section 4, and the functions $P\left(c \mid \tilde{c}, c_{v}\right)$ and $\dot{\omega}_{c}(c)$ are discussed in Section 5.

\section{Review of DNS dataset}

Previously published DNS of high Karlovitz number $n$-heptane flames are used in this study to investigate the filtered chemical source terms. The 10 simulations used were previously reported by Lapointe et al. [17] and Bobbitt et al. [20]. The reader is referred to Refs. $[15,17,18]$ for description of the numerical framework, governing equations, and validation. 


\subsection{Flow configuration}

The flow configuration used for all the simulations is illustrated in Fig. 1. It was chosen to achieve a statistically-planar, freely propagating flame without mean shear and mean curvature effects. Inflow/outflow boundary conditions are used in the $x$ direction and periodic boundary conditions are used in the $y$ and $z$ directions. The domain size is $L_{x} \times L \times L$ in $x, y$, and $z$ respectively. The domain size is related to the integral length scale with $l=0.19 L$. The simulations can be run for an unbounded time, allowing to reach a statistically-stationary state.

Since the flow configuration lacks generation of turbulence due to large scale flow straining, turbulence forcing is necessary [21-28]. The forcing magnitude is increased $0.05 L_{x}$ (where $L_{x}$ is the domain length) downstream of the inlet to reach the desired turbulent kinetic energy $(k)$ value. The velocity field forcing is stopped after a distance of $0.75 L_{x}$ and the velocity fluctuations decay rapidly, such that there are no negative axial velocities at the outlet. For each simulation, the mean inlet velocity is constant throughout the simulation and selected to match approximately the turbulent flame speed of that simulation. In practice, the flames drift slightly, but the drift is negligible compared to the domain length. The simulations are performed for at least 50 eddy turnover times, $\tau=l / u^{\prime}$, where $u^{\prime}$ is the turbulent RMS velocity and $l$ is the integral length scale, and statistics are computed using the last $30 \tau$ to remove any initial transient effects.

In the present configuration, velocity field forcing is necessary to maintain a constant turbulence intensity and achieve a statistically-stationary state. Linear velocity forcing [29-31] was chosen for its physical nature and good stability properties. The linear forcing method mimics the missing large scale straining through the ad- 
dition of a source term to the momentum equation. In recent work [20], this forcing technique was found to produce similar vorticity characteristics across the flame as the slot burner premixed flames of Sankaran et al. [16].

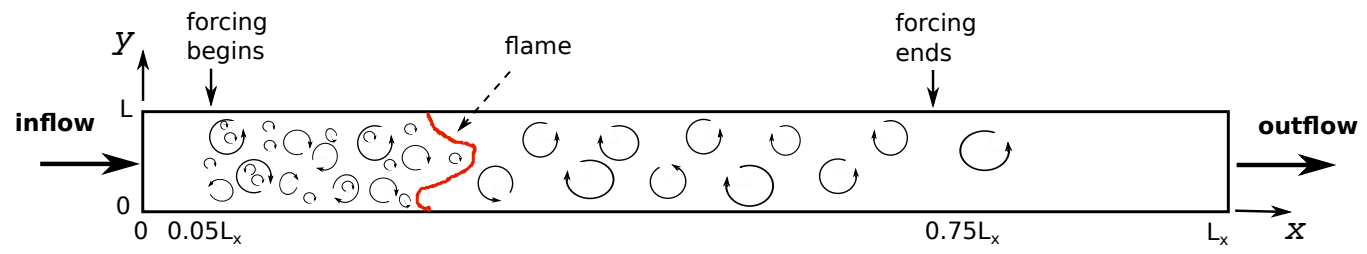

Fig. 1. Schematic diagram of the flow configuration, adapted from Savard et al. [15].

\subsection{Simulation parameters}

For each simulation, the unburnt gas is an $n$-heptane/air mixture at standard pressure $\left(P_{0}=1 \mathrm{~atm}\right)$ and equivalence ratio of 0.9 . The unburnt temperature, $T_{u}$, and turbulent RMS velocity, $u^{\prime}$, were varied to cover a range of reaction zone Karlovitz numbers. Table 1 lists the parameters for the simulations.

For each case $\left(\mathrm{A}, \mathrm{B}, \mathrm{B}^{4}, \mathrm{C}\right.$, and $\left.\mathrm{D}\right)$, detailed chemistry simulations were performed using both non-unity (no subscript) and unity Lewis numbers (subscript 1) to isolate the effects of differential diffusion. The flames were computed using constant non-unity Lewis numbers. The impact of using constant Lewis numbers as opposed to using mixture-averaged diffusion coefficients was studied in recent work $[17,32,33]$ and found to be negligible in the present $n$-heptane flames. A reduced mechanism consisting of 35 species and 217 reactions was used [18].

The focus of this study of high Karlovitz number flames is on the effects of smallscale turbulence on the chemical source terms. Given that purpose and computational cost considerations, a small integral length scale of $l / l_{F} \approx 1$ is used for most cases. The effects of integral length scale on the filtered source terms are assessed in Appendix B using cases $\mathrm{B}^{4}$ and $\mathrm{B}_{1}^{4}$ at $l / l_{F}=4$. Unfortunately, DNS at larger 


\begin{tabular}{l|ccc|cc|} 
Case & A & B & $\mathrm{B}^{4}$ & $\mathrm{C}$ & $\mathrm{D}$ \\
\hline$T_{u}(\mathrm{~K})$ & & 298 & & \multicolumn{2}{|c|}{800} \\
$S_{L}(\mathrm{~m} / \mathrm{s})$ & & 0.36 & & \multicolumn{2}{|c|}{2.3} \\
$l_{F}(\mathrm{~mm})$ & & 0.39 & & \multicolumn{2}{|c}{0.25} \\
$u^{\prime} / S_{L}$ & 9 & 18 & 33 & 19 & 45 \\
$l / l_{F}$ & 1.1 & 1.1 & 4 & 1.2 & 1.2 \\
$\mathrm{Ka}_{u}$ & 78 & 220 & 220 & 204 & 740 \\
$\mathrm{Ka}_{\delta}$ & 1.9 & 7.0 & 7.0 & 73 & 256 \\
$\mathrm{Re}_{t}$ & 83 & 190 & 1150 & 170 & 380 \\
$L(\mathrm{~m})$ & 0.0023 & 0.0023 & 0.0084 & 0.0015 & 0.0015 \\
$L_{x}$ & $11 \mathrm{~L}$ & $11 \mathrm{~L}$ & $8 \mathrm{~L}$ & $11 \mathrm{~L}$ & $11 \mathrm{~L}$ \\
$\Delta x(\mu \mathrm{m})$ & 18 & 18 & 18 & 12 & 7 \\
$\operatorname{Grid}\left(\times 10^{6}\right)$ & 23 & 23 & 675 & 23 & 117 \\
$\bar{\delta}_{F}(\mathrm{~mm})$ & & 0.165 & & 0.168 & 0.264 \\
\hline
\end{tabular}

Table 1

Parameters of the simulations. $S_{L}$ is the laminar flame speed, $l_{F}=\left(T_{b}-T_{u}\right) /|\nabla T|_{\max }$ is the laminar flame thickness, $\mathrm{Ka}_{u}=\frac{l_{F}}{S_{L}} \sqrt{\frac{u^{\prime 3}}{\nu_{u} l}}$ is the unburnt Karlovitz number, $\mathrm{Ka}_{\delta}=$ $\delta_{F}^{2} / \eta_{\delta}^{2}$ is the reaction zone Karlovitz number evaluated using the reaction zone thickness and the Kolmogorov length scale at the reaction zone, $\operatorname{Re}_{t}=u^{\prime} l / \nu$ is the turbulent Reynolds number in the unburnt gas, $L$ is the domain size in the $y$ and $z$ directions, $L_{x}$ is the domain size in the $x$ direction, $\Delta x$ is the grid spacing (uniform in all three directions), and $\bar{\delta}_{F}$ is the mean turbulent reaction zone thickness [17]. integral length scales are prohibitively expensive.

\subsection{Progress variable definition}

The progress variable is defined here as a linear combination of species mass fractions

$$
c=\sum_{i=1}^{N} b_{i} Y_{i},
$$

where $b_{i}$ is a real coefficient. In the present study, the results are obtained with $c=Y_{\mathrm{H}_{2} \mathrm{O}}+Y_{\mathrm{H}_{2}}+Y_{\mathrm{CO}}+Y_{\mathrm{CO}_{2}}$. This definition is used for consistency with previous studies of hydrocarbon flames [34-36]. It should be noted that for the 
present flames the mass fraction of $\mathrm{H}_{2}$ constitutes less than $1 \%$ of the progress variable mass fraction. The results would thus not change significantly if the progress variable definition did not include $\mathrm{H}_{2}$. Furthermore, the other species forming the progress variable have Lewis numbers relatively close to unity $\left(\mathrm{Le}_{\mathrm{H}_{2} \mathrm{O}}=0.79\right.$, $\mathrm{Le}_{\mathrm{CO}}=1.07$, and $\left.\mathrm{Le}_{\mathrm{CO}_{2}}=1.37\right)$. It should also be noted that the fuel mass fraction cannot constitute a progress variable by itself since the fuel is fully depleted well before burnt conditions are reached.

\subsection{Chemical source terms}

The effects of intense turbulence and differential diffusion on the chemical source terms of the present $n$-heptane flames have been studied previously by Savard and Blanquart [18] and Lapointe et al. [17]. Findings relevant to the present work are briefly reviewed here.

In the unity Lewis number cases, the mean source terms conditioned on the progress variable $\left\langle\dot{\omega}_{c} \mid c\right\rangle$ are close to their corresponding freely propagating, unstretched laminar flames profiles $[17,18]$ (as shown in Fig. 2a). In contrast, in the non-unity Lewis number cases, the same source terms exhibit lower values than the corresponding laminar flames (Fig. 2b) [17, 18]. Regardless of the mean values, there are large fluctuations in these chemical source terms. This is illustrated in Fig. 2c by their probability density functions at $c_{\text {peak }}$ for cases $\mathrm{B}$ and $\mathrm{B}_{1}\left(c_{\text {peak }}\right.$ is the progress variable value of peak progress variable production rate in the turbulent flame). In both unity and non-unity Lewis number cases, the PDF illustrates large fluctuations in the unfiltered chemical source terms at a fixed $c$. 


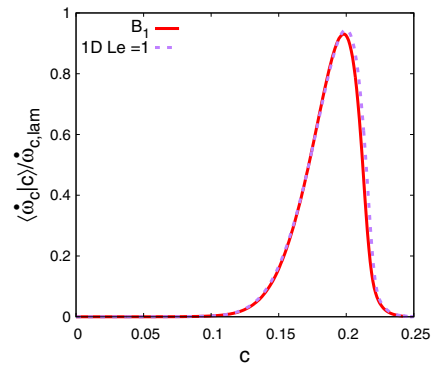

(a) Conditional mean Le $=1$

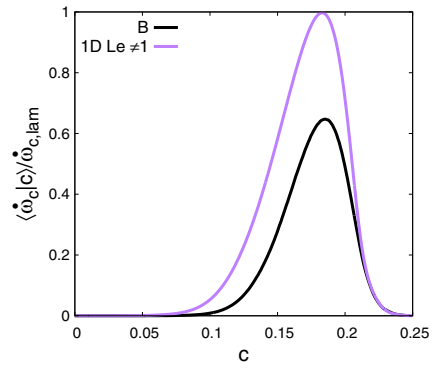

(b) Conditional mean Le $\neq 1$



(c) PDF of $\dot{\omega}_{c}$

Fig. 2. Conditional means (a-b) and probability density function (c) of the normalized progress variable production rate (normalized by the non-unity Lewis number laminar flame value). The probability density function is taken at the corresponding progress variable at peak source term.

\section{Methodology}

\subsection{Filtering of DNS data}

In the following sections, spatially filtered quantities are computed from the DNS data sets. Consider a filtering function $F$, the filtered field $\bar{\phi}$ is defined as:

$$
\bar{\phi}(\mathbf{x})=\iiint F\left(\mathbf{x}-\mathbf{x}^{\prime}\right) \phi\left(\mathbf{x}^{\prime}\right) d \mathbf{x}^{\prime}
$$

Density-weighted (Favre) filtered fields are denoted as $\tilde{\phi}$ :

$$
\tilde{\phi}=\frac{\overline{\rho \phi}}{\bar{\rho}}
$$

Practically, the filtered field (denoted by an overbar) is computed from a diffusion equation which approximates the convolution in Eq. (3) using a Gaussian filter:

$$
\bar{\phi}=\phi+\frac{\Delta^{2}}{24} \nabla^{2} \phi+\frac{\Delta^{4}}{1152} \nabla^{4} \phi,
$$

where only the first moments of the Gaussian filter are considered. A similar ap- 
proach was used by Moureau et al. [8]. Cases A through D are filtered with filter widths varying from $\Delta=4 \Delta x_{\text {DNS }}$ to $\Delta=32 \Delta x_{\text {DNS }}$. Cases $\mathrm{B}^{4}$ and $\mathrm{B}_{1}^{4}$ are filtered with larger filter widths up to $\Delta=128 \Delta x_{\text {DNS }}$. This corresponds to filter widths varying from DNS resolution to approximately six times the laminar flame thickness. Filtering the present DNS data with larger filter widths is not possible due to the low turbulent Reynolds numbers of the simulations [37].

\subsection{LES equations}

Combustion modeling using a tabulated chemistry approach is considered for the analysis of subgrid scale closures for LES. The filtered (LES) equations are as follows:

$$
\begin{gathered}
\frac{\partial \bar{\rho}}{\partial t}+\nabla \cdot(\bar{\rho} \tilde{\mathbf{u}})=0 \\
\frac{\partial \bar{\rho} \tilde{\mathbf{u}}}{\partial t}+\nabla \cdot(\bar{\rho} \tilde{\mathbf{u}} \tilde{\mathbf{u}})=-\nabla \bar{p}+\nabla \cdot \bar{\tau}+\nabla \cdot \bar{\tau}_{\mathbf{u}}^{u} \\
\frac{\partial \bar{\rho} \tilde{c}}{\partial t}+\nabla \cdot(\bar{\rho} \tilde{\mathbf{u}} \tilde{c})=\nabla \cdot \tilde{\mathbf{j}}_{c}+\nabla \cdot \bar{\tau}_{c}+\bar{\rho} \tilde{\dot{\omega}}_{c}
\end{gathered}
$$

where $\bar{\rho}, \tilde{\mathbf{u}}$, and $\tilde{c}$ are the resolved quantities, $\bar{\tau}_{\mathbf{u}}^{u}$ and $\bar{\tau}_{c}$ are subgrid-scale terms, and $\mathbf{j}_{c}$ is the diffusion flux. The progress variable is a linear combination of species mass fractions as defined in Section 2.3.

The diffusion flux is not closed in the general (non-unity Lewis number) case. In the unity Lewis number limit, the diffusion flux reduces to a simple closed form $\mathbf{j}_{c}=$ $\rho \alpha \nabla c$. The subgrid-scale terms, along with the filtered chemical source term $\tilde{\dot{\omega}}_{c}$, represent unresolved phenomena by the LES grid and require closure. The present study focuses solely on the filtered chemical source term. The treatment of the diffusion flux and the closure of the sub-filter terms are beyond the scope of this paper. 


\subsection{Comparison metrics}

In the next sections, the assumptions previously mentioned in Section 1 will be assessed using four different comparison metrics.

The filtered source terms from detailed and tabulated chemistry will be first compared through their conditional means, $\left\langle\tilde{\dot{\omega}}_{c} \mid \tilde{c}\right\rangle$, and second through their conditional probability density functions $\operatorname{PDF}\left(\tilde{\dot{\omega}}_{c} \mid \tilde{c}_{\text {peak }}\right)$. Both conditional means and PDFs are computed using volumetric weighting. In other words, computational cells whose value of $\tilde{c}$ are within a small range (bin) of the desired value are considered. The mean or probability are then computed by weighting the values of $\tilde{\dot{\omega}}_{c}$ by the corresponding cell volume.

Third, the source term tabulation will be assessed through the global prediction error made on any filtered tabulated chemical source term $\tilde{\dot{\omega}}_{\text {table }}$ compared to the filtered detailed chemistry source term $\tilde{\dot{\omega}}_{c}^{\mathrm{DKM}}[10,38]$ :

$$
\epsilon_{\text {table }}=\sqrt{\frac{\int_{\Omega}\left(\tilde{\dot{\omega}}_{\text {table }}-\tilde{\dot{\omega}}_{c}^{\text {DKM }}\right)^{2} \mathrm{~d} V}{\int_{\Omega}\left(\tilde{\dot{\omega}}_{c}^{\text {DKM }}\right)^{2} \mathrm{~d} V} .}
$$

It should be noted that $\tilde{\dot{\omega}}_{\text {table }}$ is a placeholder for any source term read from a table. This is the most strigent test since local point-wise errors can be made even if the tabulated and exact filtered source terms have similar means and PDFs. It is also a measure of how the fluctuations in the detailed chemistry source terms are captured by the tabulated source terms.

Finally, the performance of the tables will be compared through the time-averaged turbulent flame speed computed as the volumetric integral of the progress variable 
source term $[23,39]$ :

$$
S_{T}=\frac{1}{\rho_{u} c_{b} L^{2}} \int_{V} \bar{\rho} \tilde{\dot{\omega}}_{c} \mathrm{~d} V
$$

where subscripts $u$ and $b$ denote values taken in the unburnt and burnt gases, respectively, and $L$ is the domain size in the $y$ and $z$ directions (see Fig. 1). This a very important metric since accurate prediction of the turbulent flame speed is often crucial in the design of practical devices [40]. It is also the most lenient metric since local prediction errors may not have an impact on the global flame speed.

A summary of the metrics used to assess the different assumptions is provided in Table 2.

Table 2

\begin{tabular}{c|cccc|} 
Metrics & $\left\langle\tilde{\dot{\omega}}_{c} \mid \tilde{c}\right\rangle$ & $\operatorname{PDF}\left(\tilde{\dot{\omega}}_{c} \mid \tilde{c}_{\text {peak }}\right)$ & $\epsilon$ & $\bar{S}_{T}$ \\
\hline Assumption 1 & $\checkmark$ & $\checkmark$ & $\checkmark$ & \\
Assumption 2 & & & $\checkmark$ & \\
Function 1 & $\checkmark$ & & $\checkmark$ & $\checkmark$ \\
Function 2 & $\checkmark$ & $\checkmark$ & $\checkmark$ \\
\hline
\end{tabular}

Comparison metrics used for the different assumptions.

First, the filtered chemical source terms from the detailed chemistry cases will be compared to tabulated chemistry (assumption 1). Second, tabulation of the filtered source terms using $\tilde{c}$ and its variance will be assessed (assumption 2). Third, the filtered source terms will be compared to predictions from commonly used PDF models (function 1). Finally, the possibility of using laminar flames to generate the table will be discussed (function 2). 


\section{Assumptions}

\subsection{Assumption 1: Detailed vs. tabulated chemistry}

The present section is not dedicated to finding what the mean function $\left(\dot{\omega}_{c}(c)\right)$ is, nor what the fluctuations are due to, nor how to reproduce them. Instead, the focus is on whether capturing these fluctuations is necessary for LES modeling. That is assumption 1 of the introduction.

To test this assumption, results obtained with detailed chemistry (DKM) are compared to results obtained with tabulated chemistry (TAB) using a table of a single progress variable. The term "tabulated chemistry" is used in this section to refer to $\dot{\omega}_{c}(c)$. It is important to note that this source term is computed from the DNS conditional mean and not from 1D laminar flames. Throughout this section, the tabulated source term "TAB" is thus $\dot{\omega}_{c}^{\mathrm{TAB}}(c)=\left\langle\dot{\omega}_{c}^{\mathrm{DKM}} \mid c\right\rangle$. For each case A through D, the statistics of the filtered chemical source terms for detailed and tabulated chemistry are obtained as follows:

(1) Get the unfiltered source term field $\dot{\omega}_{c}^{\mathrm{DKM}}(x, y, z)$ from the detailed chemistry simulation.

(2) Compute the conditional mean $\left\langle\dot{\omega}_{c}^{\mathrm{DKM}} \mid c\right\rangle$.

(3) Tabulate the source term against the progress variable using the conditional mean $\dot{\omega}_{c}^{\mathrm{TAB}}(c)=\left\langle\dot{\omega}_{c}^{\mathrm{DKM}} \mid c\right\rangle$.

(4) Use the DKM field of $c(x, y, z)$ to lookup the tabulated source term $\dot{\omega}_{c}^{\mathrm{TAB}}(c)$ and get the field $\dot{\omega}_{c}^{\mathrm{TAB}}(x, y, z)$.

(5) Perform spatial filtering operation described in Section 3.1 to obtain $\tilde{\dot{\omega}}_{c}^{\mathrm{DKM}}(x, y, z)$ and $\tilde{\dot{\omega}}_{c}^{\mathrm{TAB}}(x, y, z)$. 
(6) Compute conditional means and conditional PDFs $\left\langle\tilde{\dot{\omega}}_{c} \mid \tilde{c}\right\rangle$ and $\operatorname{PDF}\left(\tilde{\dot{\omega}}_{c} \mid \tilde{c}_{\text {peak }}\right)$ for both DKM and TAB.

The possibility of using a table generated from 1D flamelet solutions, as an approximation for the conditional mean $\dot{\omega}_{c}^{\mathrm{TAB}}(c)$, is discussed in Section 5.2.

Figure 3 compares the filtered progress variable source term from detailed chemistry DNS, $\tilde{\dot{\omega}}_{c}^{\mathrm{DKM}}$, to tabulated chemistry, $\tilde{\dot{\omega}}_{c}^{\mathrm{TAB}}$, at different filter widths. The two conditional means $\left\langle\tilde{\dot{\omega}}_{c} \mid \tilde{c}\right\rangle$ are shown in Fig. 3a for the unity Lewis number case case $B_{1}^{4}$. Case $B_{1}^{4}$ is used since it allows filtering at larger filter widths than case $B_{1}$. As shown in Appendix B, the change in integral length scale does not significantly affect the source term. Overall, as expected, filtering reduces the magnitude of the peak source term and flattens the profile. The two conditional means are in very good agreement for all filter widths. To further illustrate the effects of filtering, the peak value of the mean filtered progress variable production rate is plotted against filter width in Fig. $3 b$ for all $\mathrm{Le}=1$ cases $\mathrm{A}$ through $\mathrm{D}$, covering a range of reaction zone Karlovitz numbers. The same decrease in the peak source term with increasing filter width is observed for all cases, independent of the chemical model (detailed vs. tabulated) and Karlovitz number. This result is important as it underscores that the fluctuations in the unfiltered source terms at a given $c$ (Fig. 2c) are not essential in predicting the mean filtered source term. It should be noted that $\Delta / \bar{\delta}_{F}$, where $\bar{\delta}_{F}$ is the mean reaction zone thickness of the turbulent flame, is preferred over $\Delta / l_{F}$ for the present flames in the thin and distributed reaction zones regimes since thickening of the preheat and reaction zones is observed. Values of $\bar{\delta}_{F}$ for the present cases were reported by Lapointe et al. [17] and are listed in Table 1.

As reproducing only the conditional mean profiles $\left(\left\langle\tilde{\dot{\omega}}_{c} \mid \tilde{c}\right\rangle\right)$ may be insufficient, the filtered source terms from detailed and tabulated chemistry are further com- 


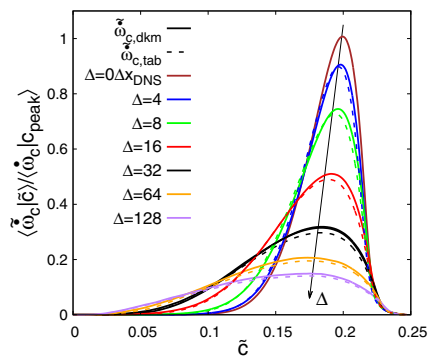

(a) Conditional means (case $\left.\mathrm{B}_{1}^{4}\right)$

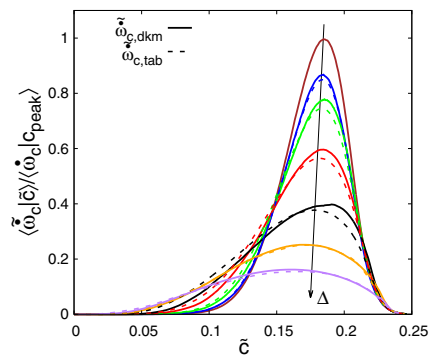

(d) Conditional means (case $\mathrm{B}^{4}$ )

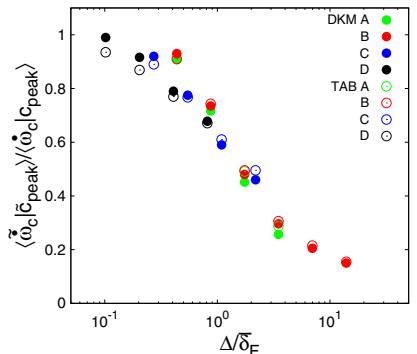

(b) Conditional means at $c_{\text {peak }}($ Le $=1)$

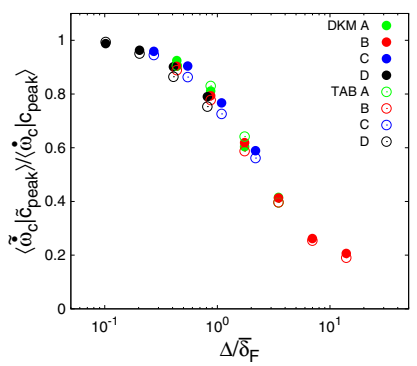

(e) Conditional means at $c_{\text {peak }}($ Le $\neq 1)$

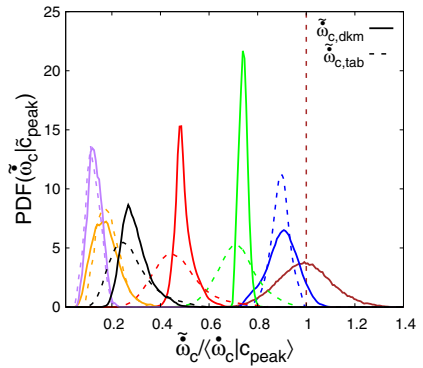

(c) PDF of $\tilde{\dot{\omega}}_{c}$ (case $\mathrm{B}_{1}^{4}$ )

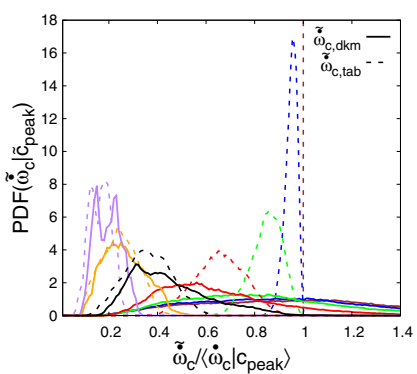

(f) PDF of $\tilde{\dot{\omega}}_{c}\left(\right.$ case B $\left.{ }^{4}\right)$

Fig. 3. Conditional means (a-d) and probability density functions (c-f) of the filtered progress variable source term from detailed chemistry DNS (solid lines) compared with filtered tabulated chemistry (dashed lines) for unity Lewis number case $\mathrm{B}_{1}^{4}$ (top) and non-unity Lewis number case $\mathrm{B}^{4}$ (bottom). b-e) Conditional means of $\tilde{\dot{\omega}}_{c}$ at $\tilde{c}_{\text {peak }}$ for cases A through D plotted against the normalized filter width $\Delta / \bar{\delta}_{F}$, where $\bar{\delta}_{F}$ is the mean reaction zone thickness of the turbulent flame [17].

pared by evaluating their probability density function on the isosurface of $\tilde{c}_{\text {peak }}$, as shown in Fig. 3c (this is the second metric discussed in Section 3.3). Fluctuations in this filtered source term can come from two different sources: (1) from the unfiltered source term at a fixed $c$ (Fig. 2c), and (2) from the spatial filtering procedure. As expected, significant discrepancies are observed at small filter widths $\Delta<l_{F}\left(\Delta \leq 16 \Delta x_{\mathrm{DNS}}\right)$. The PDF of the tabulated source terms is a delta function at $\Delta=0$ (as shown by the dashed brown lines in Figs. 3c and 3f) and gets broader as the filter width is increased. This is due to the spatial filtering procedure. Different trends in the PDFs are observed for the detailed chemistry source terms. The PDF gets thinner suggesting that the fluctuations due to turbulence-chemistry interactions are averaged out. However, at filter widths $\Delta \gtrsim l_{F}\left(\Delta \geq 32 \Delta x_{\mathrm{DNS}}\right)$, the 
PDFs of $\tilde{\dot{\omega}}_{c}$ from filtered detailed and tabulated chemistry are in decent agreement. At these larger filter widths, the flow structures affecting the chemical source terms are filtered out and only fluctuations from the spatial filtering procedure remain, reducing the differences between the two combustion models.

A similar behavior is observed in the non-unity Lewis number cases. Figures $3 \mathrm{~d}$ and 3f present the conditional means and PDFs of the filtered progress variable source terms from detailed chemistry, and non-unity Lewis number DNS (case $\mathrm{B}^{4}$ ) compared to tabulated chemistry at different filter widths. The non-unity Lewis number filtered source terms are normalized by the peak of the mean unfiltered source term (Fig. 2b). Once again, the mean filtered source terms are in good agreement (Fig. 3d) and significant discrepancies in the PDFs observed at small filter widths are reduced as $\Delta$ is increased beyond the laminar flame thickness (Fig. 3f). Furthermore, this agreement is observed independently of the Karlovitz number, as illustrated in Fig. 3e showing the peak value of the mean filtered progress variable production rate plotted against filter width. As in the unity Lewis number cases (Fig. 3b), as similar decrease is observed for all non-unity Lewis number cases.

Finally, the detailed and tabulated source terms are compared through the global prediction error computed using Eq. (9) where $\tilde{\dot{\omega}}_{\text {table }}=\tilde{\dot{\omega}}_{c}^{\mathrm{TAB}}$. The errors are listed in Table 3. These results highlight the same three conclusions. First, the error for unity Lewis number cases is less than the error for non-unity Lewis number cases. Second, the error decreases as the filter width increases. Third, the error is fairly independent of Karlovitz number for Le $=1$, but it decreases with Karlovitz number for Le $\neq 1$. This is consistent with differential diffusion effects slowly disappearing as Ka increases [17, 23, 41].

These results indicate that, while fluctuations due to turbulence affect the DKM 


\begin{tabular}{|c|cccc|cccc|}
\hline & \multicolumn{4}{|c|}{ Le $=1$} & \multicolumn{4}{c|}{ Le $\neq 1$} \\
\hline$\Delta / \Delta x_{\text {DNS }}$ & $\mathrm{A}_{1}$ & $\mathrm{~B}_{1}$ & $\mathrm{C}_{1}$ & $\mathrm{D}_{1}$ & $\mathrm{~A}$ & $\mathrm{~B}$ & $\mathrm{C}$ & $\mathrm{D}$ \\
\hline 4 & 0.16 & 0.21 & 0.24 & 0.21 & 0.58 & 0.47 & 0.36 & 0.28 \\
8 & 0.16 & 0.20 & 0.23 & 0.20 & 0.57 & 0.46 & 0.34 & 0.25 \\
16 & 0.15 & 0.19 & 0.19 & 0.18 & 0.55 & 0.42 & 0.30 & 0.22 \\
32 & 0.13 & 0.16 & 0.14 & 0.15 & 0.52 & 0.34 & 0.24 & 0.18 \\
64 & \multicolumn{3}{|c|}{0.15} & & & & 0.31 & \\
128 & \multicolumn{3}{|c|}{0.13} & & \multicolumn{3}{|c|}{0.24} \\
\hline
\end{tabular}

Table 3

Prediction error from the filtered progress variable source term from tabulated chemistry DNS compared to filtered detailed chemistry DNS for the different cases.

filtered source terms at small filter widths, their influence becomes negligible when the filter width is larger than the flame thickness. This is consistent with the fact that fluctuations in the chemical source terms are due to small turbulent scales of the order of the reaction zone thickness [17], and are thus sub-filter phenomena. In summary, assumption 1 is valid for $\Delta \gtrsim l_{F}$ when $\dot{\omega}_{c}(c)=\left\langle\dot{\omega}_{c}^{\mathrm{DKM}} \mid c\right\rangle$.

It should be noted that there might still be unsteady effects arising from sub-filter chemical source term fluctuations. Unfortunately, it is not possible to assess these effects using the present a priori analysis. A posteriori Large Eddy Simulations would need to be performed. This should be the subject of future work.

\subsection{Assumption 2: Tabulation using $\tilde{c}$ and $c_{v}$}

As discussed earlier, a common approach for LES with tabulated chemistry consists of assuming the filtered chemical source term is a function of the filtered progress variable, $\tilde{c}$, and its variance, $c_{v}$ (or, equivalently, the unmixedness factor $S_{c}$ ) $[5,11$, 42]. This is assumption 2 of the introduction. This assumption was assessed in low Karlovitz flames in recent work by Mukhopadhyay et al. [10]. The validity of such 
modeling approach for high Ka flames is assessed here by comparing the exact term to the optimal estimator given these two variables.

The optimal estimator is the function (of $\tilde{c}$ and $c_{v}$ ) that minimizes the prediction error made on the chemical source term $\left(\mathrm{L}^{2}\right.$-norm)

$$
\epsilon_{f}=\sqrt{\frac{\int_{\Omega}\left(f\left(\tilde{c}, c_{v}\right)-\tilde{\dot{\omega}}_{c}^{\mathrm{DKM}}\right)^{2} \mathrm{~d} V}{\int_{\Omega}\left(\tilde{\dot{\omega}}_{c}^{\mathrm{DKM}}\right)^{2} \mathrm{~d} V}},
$$

where $f\left(\tilde{c}, c_{v}\right)$ is any function of these two variables (note that this is simply Eq. (9) with $\left.\tilde{\dot{\omega}}_{\text {table }}=f\left(\tilde{c}, c_{v}\right)\right)$. It can be shown that the optimal estimator is exactly the conditional mean from the filtered DNS, i.e. $\left\langle\tilde{\dot{\omega}}_{c} \mid \tilde{c}, c_{v}\right\rangle$ [43]. The irreducible error associated with assuming that $\tilde{\dot{\omega}}_{c}$ is only a function of $\tilde{c}$ and $c_{v}$ is then given by Eq. (11) evaluated with $f\left(\tilde{c}, c_{v}\right)=\left\langle\tilde{\omega}_{c} \mid \tilde{c}, c_{v}\right\rangle$. These prediction errors are shown in Table 4. This is illustrated in Fig. 4 which shows the probability density function of the point-wise comparison between the predicted (optimal estimator) and the actual filtered DNS source term for $\mathrm{B}^{4}$ at three different filter widths. The prediction error, $\epsilon_{f}$, corresponds to the mean square deviation from the solid black line. The scatter around the black diagonal is limited for the three filter widths shown in Fig. 4. The correlation coefficients for the different cases are defined as

$$
r=\frac{\sum_{i=1}^{n}\left(X_{i}-\bar{X}\right)\left(Y_{i}-\bar{Y}\right)}{\sqrt{\sum_{i=1}^{n}\left(X_{i}-\bar{X}\right)^{2}} \sqrt{\sum_{i=1}^{n}\left(Y_{i}-\bar{Y}\right)^{2}}}
$$

and are shown on Fig. 4. This indicates a good prediction of the filtered source term.

For all unity Lewis number detailed chemistry cases and filter widths, the prediction error from the optimal estimator is small (at most 16\%). This indicates that, even with large source term fluctuations (Fig. 2c), a tabulation approach using only 


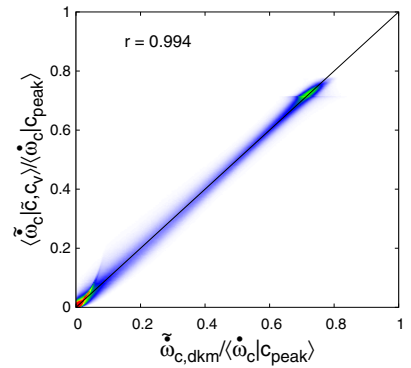

(a) $\mathrm{B}_{1}^{4}, \Delta=8 \Delta x_{\mathrm{DNS}}$

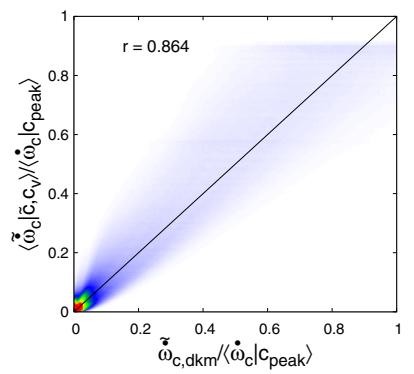

(d) $\mathrm{B}^{4}, \Delta=8 \Delta x_{\mathrm{DNS}}$

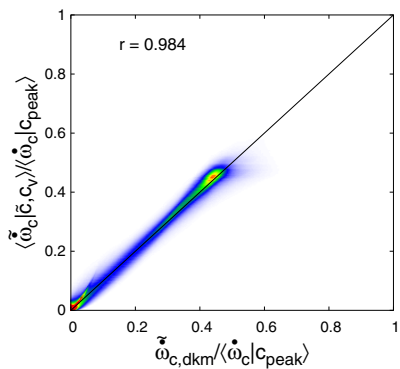

(b) $\mathrm{B}_{1}^{4}, \Delta=16 \Delta x_{\mathrm{DNS}}$

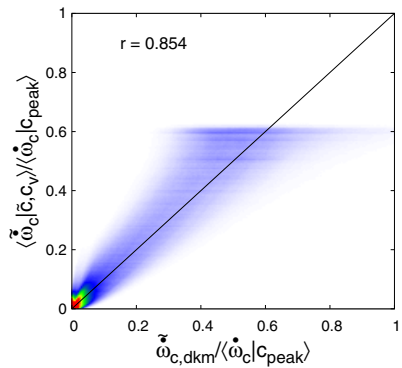

(e) $\mathrm{B}^{4}, \Delta=16 \Delta x_{\mathrm{DNS}}$



(c) $\mathrm{B}_{1}^{4}, \Delta=32 \Delta x_{\mathrm{DNS}}$

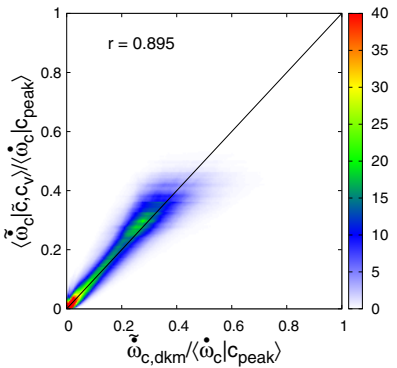

(f) $\mathrm{B}^{4}, \Delta=32 \Delta x_{\mathrm{DNS}}$

Fig. 4. Filtered chemical source obtained from the optimal estimator compared to the source term from the filtered detailed chemistry DNS for unity Lewis number case $\mathrm{B}_{1}^{4}(\mathrm{a}-\mathrm{c})$ and non-unity Lewis number case $\mathrm{B}^{4}$ (d-f) for three different filter widths. The corresponding correlation coefficient is listed.

the filtered progress variable and its variance provides a very good estimate of the filtered source term for LES of unity Lewis number flames independent of the filter width. While assumption 2 is clearly valid for these cases, it appears from the results of Table 4 that $\tilde{c}$ and $c_{v}$ are not sufficient to capture fluctuations due to differential diffusion. A third tabulation variable would be necessary.

It was shown in recent DNS of high Karlovitz number flames [33] that, in unity Lewis number cases, two variables are needed to capture the chemical source term fluctuations, namely the progress variable and its dissipation rate (i.e. $\dot{\omega}_{c} \approx \dot{\omega}_{c}(c, \chi)$ where $\chi=2 \alpha|\nabla c|^{2}$ ). In non-unity Lewis number flames, a third variable, the diffusion rate $\xi=\nabla \cdot(\rho \alpha \nabla c)$, is needed to accurately capture the chemical source term fluctuations (i.e. $\dot{\omega}_{c} \approx \dot{\omega}_{c}(c, \chi, \xi)$ ).

Let's recall that, at small filter widths, the variance is modeled accurately by the 
algebraic expression $c_{v} \approx \frac{1}{12} \Delta x^{2}|\nabla \tilde{c}|^{2}$ [44]. This expression presents the same dependence on the gradient of the progress variable as the dissipation rate. Therefore, in the limit of small filter widths, the variance is analoguous to the dissipation rate and thus will lead to better predictions of the source term fluctuations. Filtering might affect the dependence of the source term on $c$ and $\chi$ but the trends should still be present. Given this observation and the results from Ref. [33], it is expected that assumption 2 would lead to smaller errors than assumption 1 for unity Lewis number cases. This is confirmed by comparing values in Tables 3 and 4 . More specific to the present discussion, it also explains the larger errors for non-unity Lewis number flames. The variance is not sufficient to capture the effects of both $\chi$ and $\xi$.

However, fluctuations due to differential diffusion are caused by curvature at small scales of the order of the reaction zone thickness [18] and are thus significantly reduced when $\Delta \geq l_{F}$. The filtered progress variable and its variance are then sufficient to tabulate the filtered source term for non-unity Lewis number flames at large filter widths as the error is reduced and approaches that of unity Lewis number flames.

\begin{tabular}{|c|cccc|cccc|}
\hline & \multicolumn{4}{|c|}{ Le $=1$} & \multicolumn{4}{c|}{ Le $\neq 1$} \\
\hline$\Delta / \Delta x_{\text {DNS }}$ & $\mathrm{A}_{1}$ & $\mathrm{~B}_{1}$ & $\mathrm{C}_{1}$ & $\mathrm{D}_{1}$ & $\mathrm{~A}$ & $\mathrm{~B}$ & $\mathrm{C}$ & $\mathrm{D}$ \\
\hline 4 & 0.06 & 0.07 & 0.12 & 0.15 & 0.44 & 0.31 & 0.32 & 0.26 \\
8 & 0.04 & 0.06 & 0.12 & 0.13 & 0.37 & 0.30 & 0.28 & 0.22 \\
16 & 0.06 & 0.09 & 0.12 & 0.12 & 0.37 & 0.29 & 0.21 & 0.17 \\
32 & 0.11 & 0.13 & 0.13 & 0.11 & 0.34 & 0.22 & 0.15 & 0.12 \\
64 & 0.15 & & & 0.25 & & \\
128 & 0.16 & & & 0.28 & \\
\hline
\end{tabular}

Table 4

Prediction error from the optimal estimator of the progress variable source term for detailed chemistry simulations for the different cases. 


\section{Unknown functions}

\subsection{Presumed sub-filter PDF}

In Sections 4.1 and 4.2, it was shown that it is not necessary to capture the fluctuations in the unfiltered chemical source terms (i.e. $\left.\dot{\omega}_{c} \approx \dot{\omega}_{c}(c)\right)$ and that $\tilde{c}$ and $c_{v}$ are good tabulation variables for the filtered chemical source term (i.e. $\tilde{\dot{\omega}}_{c} \approx \tilde{\dot{\omega}}_{c}\left(\tilde{c}, c_{v}\right)$ ). In this section, the filtered source terms predicted from $\beta$-PDF, and FLF-PDF closures (defined in Appendix A) are compared to the filtered DNS in order to determine an appropriate function for $P\left(c \mid \tilde{c}, c_{v}\right)$.

\subsubsection{Comparison of the sub-filter PDF}

In order to assess the validity of common presumed-PDF models, it is insightful to first evaluate the sub-filter PDF from the filtered DNS. Figure 5 presents the subfilter PDFs of $c$ computed at a fixed $\tilde{c}=0.12$ for two values of $S_{c}$ for the different cases. A value of 0.12 was chosen since it corresponds to the midpoint between the unburnt (0) and burnt (0.24) values. The sub-filter PDFs were also compared at values of $\tilde{c}=0.06$ and $\tilde{c}=0.18$ and similar trends were observed.

First, it should be noted that the sub-filter PDFs of the filtered detailed chemistry DNS are not significantly influenced by differential diffusion effects or changes in integral length scale. This is illustrated in Fig. 5a. Second, the shape of the subfilter PDFs from the filtered DNS are in better agreement with the FLF-PDF than the $\beta$-PDF (Figs. 5b and 5c). This agrees with the results of Moureau et al. [8] and Mukhopadhyay et al. [10] at lower Karlovitz numbers. However, larger differences are observed here between the FLF-PDF and filtered DNS PDF. This is likely due 

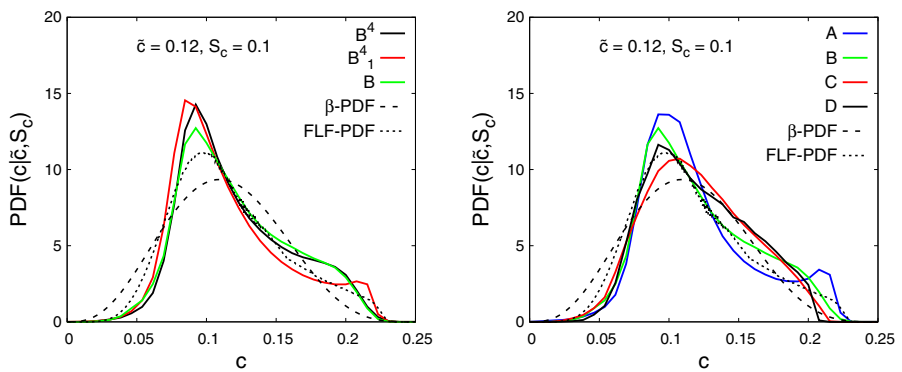

(a) $\tilde{c}=0.12, S_{c}=0.1$

(b) $\tilde{c}=0.12, S_{c}=0.1$

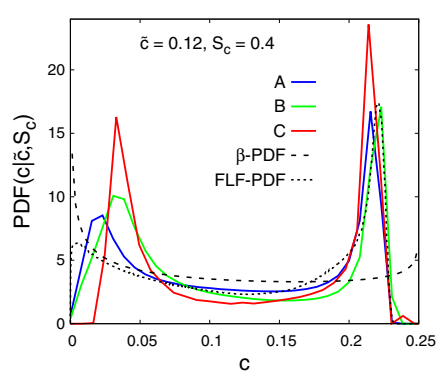

(c) $\tilde{c}=0.12, S_{c}=0.4$

Fig. 5. Sub-filter probability density function of $c$ at given $\tilde{c}$ and $S_{\tilde{c}}$ comparing filtered detailed chemistry DNS, $\beta$-PDF, and FLF-PDF. The DNS are filtered with $\Delta=32 \Delta x_{\text {DNS }}$.

to the much higher Karlovitz numbers of the present simulations.

Two main reasons can explain these differences in the sub-filter PDFs: (1) the turbulent flame is significantly thickened and (2) the flow field is highly threedimensional. While it is difficult to account for the shape of the turbulent flame in 1D laminar flames, the thickening of the preheat zone can be modeled through an enhanced turbulent diffusivity. Consider, for example, the semi-empirical model of Savard and Blanquart [41] for the turbulent diffusivity:

$$
D+D_{T}=D(1+a \mathrm{Ka})
$$

where $D$ is the laminar diffusivity, $D_{T}$ is the turbulent diffusivity, $a$ is a constant (a value of 0.05 is used here as in Ref. [41]), and Ka is the Karlovitz number. Note that the Karlovitz number is computed using the local, temperature-dependent, viscosity and is varied by increasing $u^{\prime}$. It is therefore higher in the unburnt gases and 
decreases across the flame, $\mathrm{Ka}=\mathrm{Ka}_{u} \sqrt{\frac{\nu_{u}}{\nu}}$. The effect of enhanced diffusivity on the FLF-PDF is studied by performing 1D laminar flame simulations with added diffusivity. The laminar diffusivity is increased following Eq. (13) over a range of unburnt Karlovitz numbers. The results are illustrated in Fig. 6a. The left peak of the PDF shifts towards the right as the Karlovitz is increased while the position of the right peak is essentially unchanged. This shift is consistent with the trend observed in the filtered DNS (Fig. 6b) from case A to case C. This can be associated with the significant thickening of the preheat zone which occurs as a result of the added turbulent diffusivity. The height of the left peak is still underpredicted by the modified FLF-PDF. This is likely due to the three-dimensional nature of the flow field.

While the shapes of the predicted PDF differ somewhat from the filtered DNS PDF, the impact of these differences on the filtered source term might be limited. This is investigated next.

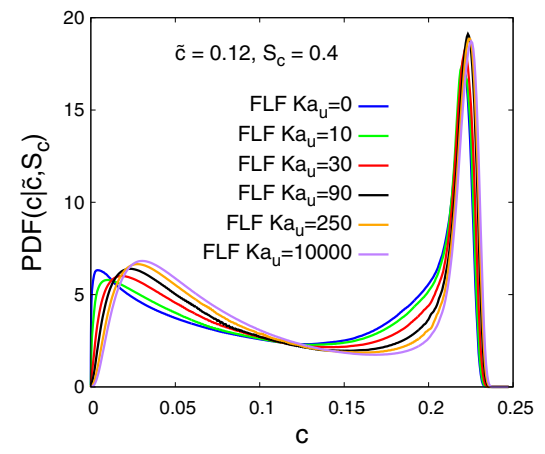

(a) FLF-PDF

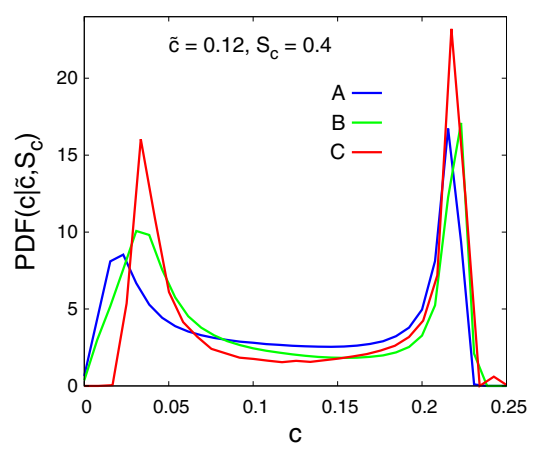

(b) Filtered DNS PDF

Fig. 6. Sub-filter probability density function of $c$ at given $\tilde{c}$ and $S_{\tilde{c}}$ comparing FLF-PDF with added diffusivity. The unburnt Karlovitz number is reported in the legend since the Karlovitz number varies across the flame due to viscosity changes. 


\subsubsection{Comparison of source terms}

The chemical source terms predicted by the $\beta$-PDF and FLF-PDF tables are compared to the filtered DNS source terms. The filtered tabulated source terms are obtained as follows:

(1) Get the unfiltered tabulated source term using the conditional mean from the DNS, $\dot{\omega}_{c}^{\mathrm{TAB}}(c)=\left\langle\dot{\omega}_{c}^{\mathrm{DKM}} \mid c\right\rangle$.

(2) Compute the filtered source term using Eq. (1), $\tilde{\dot{\omega}}_{c}^{\mathrm{TAB}}\left(\tilde{c}, c_{v}\right)=\int_{0}^{c_{b}} \dot{\omega}_{c}^{\mathrm{TAB}}(c) P\left(c \mid \tilde{c}, c_{v}\right) \mathrm{d} c$.

(3) Tabulate the filtered source term $\tilde{\dot{\omega}}_{c}^{\mathrm{TAB}}\left(\tilde{c}, c_{v}\right)$ against $\tilde{c}$ and $c_{v}$ for both the $\beta$ PDF and FLF-PDF.

(4) Use the filtered DKM fields of $\tilde{c}$ and $c_{v}$ to lookup $\tilde{\dot{\omega}}_{c}^{\mathrm{TAB}}\left(\tilde{c}, c_{v}\right)$.

Figure 7 shows the conditional means of the filtered chemical source terms obtained with the $\beta$-PDF and FLF-PDF tables for cases $\mathrm{B}^{4}$ and $\mathrm{B}_{1}^{4}$ (the conditional mean obtained using the optimal estimator is identical to the filtered DNS and is not shown). At small filter widths ( $\Delta=4$ or $8 \Delta x_{\mathrm{DNS}}$ ), the predicted source term is very close to that of the filtered DNS, regardless of the sub-filter PDF used. At higher filter widths ( $\Delta \geq 16 \Delta x_{\mathrm{DNS}}$ ), the shape of the conditional mean predicted from the table differs from the filtered DNS. This is the case for both sub-filter PDFs, but the FLF-PDF table is in overall better agreement with the filtered DNS. In all cases, the magnitude of the peak filtered source term from the PDF models agrees fairly well with the filtered DNS. Errors in $\left\langle\tilde{\dot{\omega}}_{c} \mid \tilde{c}\right\rangle$ can be due to both the convolution of the unfiltered source term with the sub-filter PDFs as well as integration over all variance values. The slightly better results obtained here with the FLF-PDF could be due to cancellation of errors.

While the conditional means of the chemical source terms $\left(\left\langle\tilde{\dot{\omega}}_{c} \mid \tilde{c}\right\rangle\right)$ are relatively well predicted by the $\beta$-PDF and FLF-PDF, it is not clear that the fluctuations in 


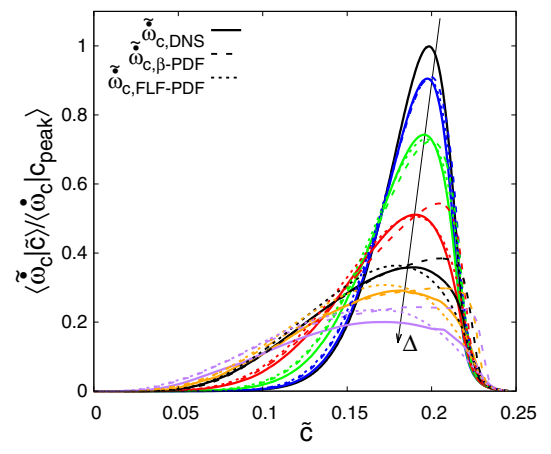

(a) Case $\mathrm{B}_{1}^{4}$

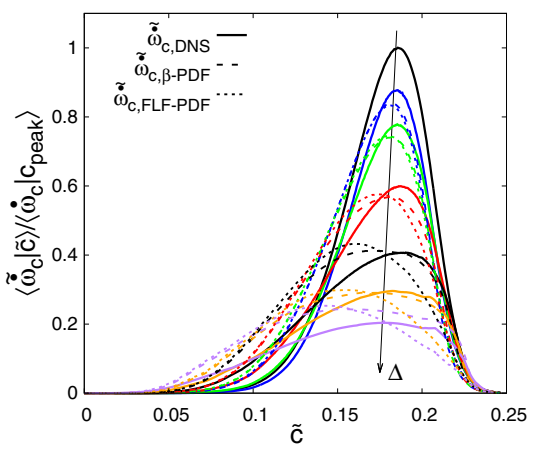

(b) Case $B^{4}$

Fig. 7. Filtered progress variable source term (solid lines) (case $\mathrm{B}^{4}$ ) compared with predictions from the $\beta$-PDF and FLF-PDF tables (dashed lines) using different filter widths $\Delta=1,4,8,16,32,64$, and $128 \Delta x_{\text {DNS }}$.

$\tilde{\dot{\omega}}_{c}$ will be captured adequately. Since the shape and location of the peaks of the conditional means differ between the filtered DNS, $\beta$-PDF, and FLF-PDF, it is not relevant to compare the probability density function of $\tilde{\dot{\omega}}_{c}$ at $\tilde{c}_{\text {peak }}$ as was done for assumption 1. Instead, as done for assumption 2, the prediction errors from the assumed PDF models, defined in Eq. (9) with $\tilde{\dot{\omega}}_{\text {table }}=\tilde{\dot{\omega}}_{c}^{\mathrm{TAB}}\left(\tilde{c}, c_{v}\right)$, are summarized in Table 5. The prediction errors are larger than for the optimal estimator up to a factor of 2 or 3 for larger filter widths. From these results, it is difficult to determine which PDF is better, as the prediction errors from both $\beta$-PDF and FLF-PDF tables are relatively close. It is also difficult to determine if the predictions from these tables are satisfactory since the mean source terms are relatively well predicted but significant point-wise errors are observed.

As a final comparison, the turbulent flame speeds predicted from both $\beta$-PDF and FLF-PDF are compared in Table 6. This instantaneous flame speed is computed for each data file saved (approximately one eddy turnover time apart) using Eq. (10) and then averaged (over time) to obtain the mean turbulent flame speed $\overline{S_{T}}$. Again, the filtered progress variable and its variance are evaluated from the (filtered) DNS and are used to lookup the filtered density and filtered source terms from the $\beta$ - 


\begin{tabular}{|c|c|c|c|c|c|c|c|c|c|}
\hline & & & $\mathrm{Le}=$ & $=1$ & & & $\mathrm{Le}$ & $\neq 1$ & \\
\hline & $\Delta / \Delta x_{\mathrm{DNS}}$ & $\mathrm{A}_{1}$ & $\mathrm{~B}_{1}$ & $\mathrm{C}_{1}$ & $\mathrm{D}_{1}$ & A & B & $\mathrm{C}$ & D \\
\hline & 4 & 0.17 & 0.25 & 0.27 & 0.22 & 0.58 & 0.50 & 0.52 & 0.55 \\
\hline & 8 & 0.18 & 0.26 & 0.26 & 0.20 & 0.58 & 0.48 & 0.50 & 0.54 \\
\hline$\rho$ & 16 & 0.28 & 0.31 & 0.25 & 0.18 & 0.53 & 0.43 & 0.46 & 0.52 \\
\hline & 32 & 0.49 & 0.49 & 0.31 & 0.17 & 0.41 & 0.35 & 0.41 & 0.50 \\
\hline & 64 & & 0.55 & & & & 0.36 & & \\
\hline & 128 & & 0.57 & & & & 0.40 & & \\
\hline & 4 & 0.18 & 0.25 & 0.27 & 0.23 & 0.58 & $0.4 C$ & 0.52 & 0.55 \\
\hline & 8 & 0.19 & 0.27 & 0.26 & 0.21 & 0.58 & 0.49 & 0.51 & 0.54 \\
\hline ГI & 16 & 0.20 & 0.27 & 0.24 & 0.20 & 0.55 & 0.45 & 0.50 & 0.53 \\
\hline & 32 & 0.21 & 0.29 & 0.22 & 0.18 & 0.46 & 0.42 & 0.48 & 0.53 \\
\hline & 64 & & 0.30 & & & & 0.42 & & \\
\hline & 128 & & 0.33 & & & & 0.45 & & \\
\hline
\end{tabular}

Table 5

Prediction error (Eq. (9)) from $\beta$-PDF, and FLF-PDF tables of the progress variable source term for the different cases.

PDF and FLF-PDF tables. As shown in Table 6 and in all cases, both $\beta$-PDF and FLF-PDF predict the turbulent flame speed accurately (within a few \%). This result remains valid even for the non-unity Lewis number cases. This is in contrast to the large point-wise errors reported in Table 5, and suggests that an accurate prediction of the mean filtered source term is sufficient to obtain a good estimate of the turbulent flame speed.

In summary, the shape of the FLF-PDF is in better agreement with the filtered DNS. However, both the FLF-PDF and $\beta$-PDF models lead to similar point-wise errors on the source terms and similar turbulent flame speeds. The present results suggest that further work should be done to improve sub-filter PDF models for high Karlovitz number flows. 


\begin{tabular}{|c|c|cccc|cccc|}
\hline \multicolumn{2}{|c|}{} & \multicolumn{5}{|c|}{ Le $=1$} & \multicolumn{4}{|c|}{ Le $\neq 1$} \\
\hline & $\Delta / \Delta x_{\text {DNS }}$ & $\mathrm{A}_{1}$ & $\mathrm{~B}_{1}$ & $\mathrm{C}_{1}$ & $\mathrm{D}_{1}$ & $\mathrm{~A}$ & $\mathrm{~B}$ & $\mathrm{C}$ & $\mathrm{D}$ \\
\hline \multirow{5}{*}{$\beta$} & 4 & 1.00 & 1.00 & 0.99 & 1.00 & 0.99 & 0.98 & 0.93 & 0.94 \\
& 8 & 0.99 & 0.99 & 0.99 & 1.00 & 0.99 & 0.98 & 0.93 & 0.94 \\
& 16 & 1.00 & 1.00 & 0.99 & 1.00 & 0.99 & 0.99 & 0.95 & 0.95 \\
& 32 & 1.05 & 1.03 & 1.02 & 1.02 & 1.03 & 1.08 & 1.00 & 0.96 \\
& 64 & & 1.02 & & & & 1.10 & & \\
& 128 & & 1.05 & & & & 1.12 & & \\
\hline \multirow{5}{*}{ FLF } & 4 & 1.05 & 1.01 & 1.00 & 1.02 & 1.01 & 1.01 & 0.97 & 1.01 \\
& 8 & 1.06 & 0.99 & 1.01 & 1.01 & 1.01 & 1.00 & 0.96 & 1.01 \\
& 16 & 0.99 & 1.00 & 1.00 & 1.01 & 1.00 & 1.01 & 0.98 & 1.01 \\
& 32 & 1.01 & 1.01 & 1.01 & 1.00 & 1.02 & 1.06 & 1.01 & 1.02 \\
& 64 & & 1.02 & & & & 1.08 & & \\
& 128 & & 1.01 & & & & 1.09 & & \\
\hline
\end{tabular}

Table 6

Predicted turbulent flame speeds ( $S_{T}$ from Eq. (10)) from $\beta$-PDF and FLF-PDF tables for the different cases normalized by the actual flame speed.

5.2 Unfiltered chemical source term $\dot{\omega}_{c}(c)$

Throughout the previous analysis, the mean chemical source terms from the detailed chemistry DNS were used to generate the chemistry tables, in other words $\dot{\omega}_{c}^{\mathrm{TAB}}(c)=\left\langle\dot{\omega}_{c}^{\mathrm{DKM}} \mid c\right\rangle$. However, in practice, this function is unknown and it is desirable to generate it a priori using 1D flamelet solutions.

As shown in Fig. 2a, in the unity Lewis number case, the mean progress variable source term is well approximated by the corresponding one-dimensional, unstretched laminar flame. Thus, laminar flames can be readily used to populate chemistry tables for LES. While assuming unity Lewis numbers may be unrealistic for heavy fuels such as $n$-heptane, it could apply to fuels with close to unity Lewis numbers such as methane. In recent work [39], it was shown that the reduction in the chemical source terms due to differential diffusion effects was much lesser for 
methane than for heavy hydrocarbon fuels with non-unity Lewis numbers.

For fuels with non-unity Lewis numbers, it was shown in previous work [17, 18] that the mean fuel consumption rate (and, similarly, the mean progress variable source term) is lower than in the corresponding unity Lewis number flame (see Fig. 2b). This was observed for different fuels, equivalence ratios, and chemical mechanisms [39]. This reduction in fuel consumption was previously related to differential diffusion effects and turbulent mixing. Savard and Blanquart [18] showed that, in the present case B, low fuel consumption rates are correlated with regions of high curvature. This is observed for moderate Karlovitz numbers cases A and B. However, as the Karlovitz number is further increased, as in cases C and D, differential diffusion effects are diminished with increasing turbulent mixing (effective Lewis numbers tend towards unity as the Karlovitz number increases $[17,41])$. The chemical source terms are then less sensitive to curvature and the effective mean values increase towards the unity Lewis number values. This is illustrated in Fig. 8 presenting the peak values of the mean unfiltered progress variable production rate for cases A through D.

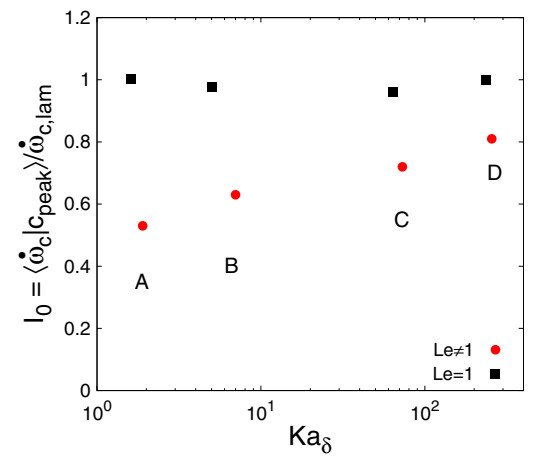

Fig. 8. Mean progress variable source term at $c_{\text {peak }}$ for simulations at varying reaction zone Karlovitz numbers.

This suggests that, in the limit of very high Karlovitz numbers $\left(\mathrm{Ka}_{\delta}>100\right)$, a tabulation using unity Lewis number unstretched laminar flames should produce good results since turbulent mixing reduces differential diffusion effects. At the other 
extreme, for low Karlovitz number flames (flamelet regimes, $\mathrm{Ka}_{\delta}<0.1$ ), previous studies suggest that the chemical source terms can be tabulated using non-unity Lewis number unstretched laminar flames $[2,3]$. The most challenging regime (and subject of the present work) corresponds to the moderate/high Karlovitz number range, $\mathrm{Ka}_{\delta} \sim \mathcal{O}(0.1-100)$, where the mean chemical source term is not predicted as accurately by 1D unstretched laminar flames (see Fig. 8). Predicting this deviation is an active area of research and there does not currently exist a predictive model which includes differential diffusion, turbulent mixing, strain, and curvature effects in tabulated chemistry of turbulent flame simulations.

However, the mean chemical source term could be obtained by simply scaling the laminar source term by a burning efficiency:

$$
I_{0}=\frac{\left\langle\dot{\omega}_{c}^{\text {DKM }} \mid c_{\text {peak }}\right\rangle}{\dot{\omega}_{c, \text { lam }}\left(c_{\text {peak }}\right)} .
$$

This burning efficiency is not known a priori but can be estimated from DNS of turbulent flames. It was shown by Lapointe and Blanquart [39] that, at a fixed reaction zone Karlovitz number and fuel Lewis number, the burning efficiency was not affected by fuel chemistry, chemical mechanism, or equivalence ratio.

The potential of this approach is assessed by comparing tables generated by convoluting the unstretched laminar flames with the presumed PDFs for all the non-unity Lewis number cases. Two tables are built for each presumed PDF. One is built using the laminar source term and another is built with the laminar source term scaled by the burning efficiency (taken from Fig. 8).

As done with the previous assumptions, the prediction errors resulting from convoluting the laminar source term with the presumed PDFs are summarized in Table 7. 
The prediction errors are significantly larger when the burning efficiency is neglected (laminar source term, or equivalently $I_{0}=1$ ). The error is reduced when the burning efficiency is taken into account $\left(\dot{\omega}_{c, \text { lam }} \cdot I_{0}\right)$, resulting in errors very close to those reported in Table 5 using the conditional mean from the DNS.

\begin{tabular}{|c|c|c|c|c|c|c|c|c|c|}
\hline & & & $\dot{\omega}_{c}$ & lam & & & $\dot{\omega}_{c, \text { lan }}$ & $\mathrm{n} \cdot I_{0}$ & \\
\hline & $\Delta / \Delta x_{\mathrm{DNS}}$ & A & B & $\mathrm{C}$ & $\mathrm{D}$ & A & B & $\mathrm{C}$ & $\mathrm{D}$ \\
\hline \multirow{6}{*}{$\beta$} & 4 & 1.03 & 0.81 & 0.49 & 0.34 & 0.59 & 0.50 & 0.53 & 0.55 \\
\hline & 8 & 1.05 & 0.81 & 0.45 & 0.31 & 0.58 & 0.49 & 0.51 & 0.53 \\
\hline & 16 & 1.07 & 0.81 & 0.41 & 0.26 & 0.54 & 0.44 & 0.46 & 0.52 \\
\hline & 32 & 1.13 & 0.92 & 0.44 & 0.25 & 0.42 & 0.35 & 0.42 & 0.51 \\
\hline & 64 & & 0.96 & & & & 0.37 & & \\
\hline & 128 & & 1.04 & & & & 0.40 & & \\
\hline \multirow{6}{*}{ FLF } & 4 & 1.01 & 0.80 & 0.49 & 0.34 & 0.59 & 0.50 & 0.53 & 0.56 \\
\hline & 8 & 1.06 & 0.82 & 0.46 & 0.32 & 0.59 & 0.50 & 0.52 & 0.54 \\
\hline & 16 & 1.07 & 0.82 & 0.44 & 0.29 & 0.55 & 0.45 & 0.50 & 0.54 \\
\hline & 32 & 1.05 & 0.91 & 0.46 & 0.28 & 0.46 & 0.42 & 0.48 & 0.53 \\
\hline & 64 & & 0.94 & & & & 0.44 & & \\
\hline & 128 & & 0.98 & & & & 0.46 & & \\
\hline
\end{tabular}

Table 7

Prediction error from $\beta$-PDF and FLF-PDF with and without scaling by the burning efficiency tables for the different non-unity Lewis number cases.

Finally, the mean turbulent flame speeds predicted from both $\beta$-PDF and FLF-PDF with and without this scaling are compared in Table 8. Large errors in the mean turbulent flame speed are observed when "unscaled" laminar flames are used. As expected, this error decreases with increasing Karlovitz number as the burning efficiency approaches unity. Once again, the flame speed prediction is greatly improved by scaling the laminar source term by the burning efficiency. Small errors are observed in all cases and the results are very close to those obtained using the conditional mean from the DNS (Table 6). This illustrates that laminar flames can be used to generate chemistry tables given an estimate of the burning efficiency. 


\begin{tabular}{|c|c|c|c|c|c|c|c|c|c|}
\hline & & & $\dot{\omega}_{c, 1}$ & & & & $\dot{\omega}_{c, \text { lan }}$ & $\mathrm{n} \cdot I_{0}$ & \\
\hline & $\Delta / \Delta x_{\mathrm{DNS}}$ & A & B & $\mathrm{C}$ & $\mathrm{D}$ & A & B & $\mathrm{C}$ & $\mathrm{D}$ \\
\hline & 4 & 1.88 & 1.64 & 1.28 & 1.15 & 1.01 & 1.00 & 0.97 & 0.95 \\
\hline & 8 & 1.87 & 1.64 & 1.29 & 1.14 & 1.01 & 1.00 & 0.97 & 0.95 \\
\hline & 16 & 1.86 & 1.66 & 1.31 & 1.16 & 1.01 & 1.01 & 0.99 & 0.95 \\
\hline & 32 & 1.97 & 1.82 & 1.38 & 1.17 & 1.05 & 1.09 & 1.04 & 0.97 \\
\hline & 64 & & 1.84 & & & & 1.10 & & \\
\hline & 128 & & 1.85 & & & & 1.11 & & \\
\hline & 4 & 1.94 & 1.69 & 1.34 & 1.21 & 1.02 & 1.03 & 1.01 & 1.00 \\
\hline & 8 & 1.93 & 1.68 & 1.34 & 1.21 & 1.02 & 1.02 & 1.00 & 1.01 \\
\hline EI E & 16 & 1.90 & 1.68 & 1.35 & 1.22 & 1.01 & 1.03 & 1.04 & 1.01 \\
\hline & 32 & 1.92 & 1.78 & 1.39 & 1.21 & 1.04 & 1.08 & 1.05 & 1.01 \\
\hline & 64 & & 1.82 & & & & 1.10 & & \\
\hline & 128 & & 1.85 & & & & 1.11 & & \\
\hline
\end{tabular}

Table 8

Predicted turbulent flame speeds ( $S_{T}$ from Eq. (10)) from $\beta$-PDF and FLF-PDF with and without scaling by the burning efficiency tables for the different non-unity Lewis number cases normalized by the actual flame speed.

\section{Summary and conclusion}

DNS with detailed chemistry and unity and non-unity Lewis numbers were used to assess the effects of turbulence-chemistry interactions and differential diffusion effects on the sub-filter closure of the chemical source terms.

The findings are summarized in Fig. 9 by comparing the prediction errors (Eq. (9)) associated with the different assumptions (A1 and A2) and unknown functions (F1 and $\mathrm{F} 2$ ) for case $\mathrm{B}^{4}$ (both unity and non-unity Lewis numbers).

First, it was observed that filtered source terms from detailed chemistry and tabulated chemistry were in good agreement at suficiently large filter widths ( $\left.\Delta \gtrsim l_{F}\right)$ for both unity and non-unity Lewis number cases. The associated error (A1, blue 


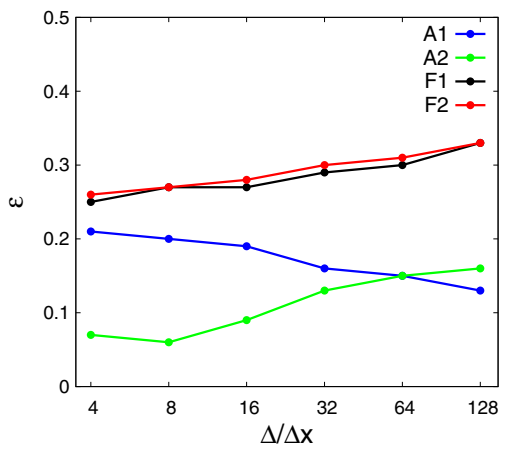

(a) $\mathrm{Le}=1\left(\right.$ case $\left.\mathrm{B}_{1}^{4}\right)$

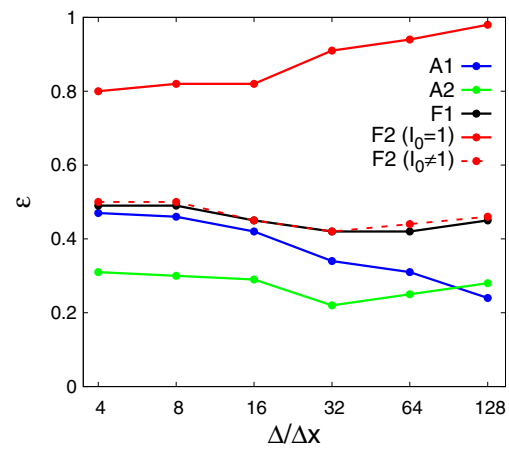

(b) Le $\neq 1\left(\right.$ case $\left.\mathrm{B}^{4}\right)$

Fig. 9. Summary of prediction errors from the assumptions (A1 and A2) and unknown functions (F1 and F2) for unity (left) and non-unity (right) Lewis number cases. Results obtained with the FLF-PDF are shown for unknown functions F1 and F2. Similar results were obtained with the $\beta$-PDF, as seen in Tables 5 and 7.

lines on Fig. 9) is greater for non-unity Lewis number cases but it decreases with increasing filter width. This seems to indicate that fluctuations in the chemical source terms observed in the detailed chemistry cases do not have a significant influence on the filtered source term and can be neglected in LES.

Second, it was shown that a tabulation approach using $\tilde{c}$ and its variance, $c_{v}$, can predict accurately the filtered chemical source terms of detailed chemistry filtered DNS. The error resulting from this assumption (A2, green lines on Fig. 9) is the irreducible error associated with assuming that $\tilde{\dot{\omega}}_{c}$ is only a function of $\tilde{c}$ and $c_{v}$. The errors from the two unknown functions (F1 and F2) will therefore be greater than the error from A2.

Third, predictions of the chemical source term from commonly assumed $\beta$-PDF and FLF-PDF models were compared to the filtered DNS. Both models lead to non-negligible errors in the local source terms (F1, black lines on Fig. 9). The error is significantly larger than the irreducible error from assumption 2. However, the mean turbulent flame speed was still predicted accurately. This is an important result since accurate prediction of the burning velocity is of importance for many 
combustion devices.

Fourth, the possibility of using 1D laminar flames to generate the chemistry tables was discussed. For heavy hydrocarbon flames $\left(\operatorname{Le}_{F}>1\right)$ at moderate/high Karlovitz numbers, the reduction in the mean chemical source term due to differential diffusion effects must be taken into account in flamelet models to predict accurately the mean turbulent flame speed. This can be done through a simple scaling of the laminar source term by a burning efficiency. The error made by neglecting the burning efficiency (F2, red lines on Fig. 9, computed using the FLF-PDF) is significantly larger than the errors associated with assumptions 1 and 2 and the presumed sub-filter PDFs.

These a priori results illustrate the potential of using simple tabulated chemistry approaches originally developed for low Karlovitz number flames to perform LES of premixed flames in the thin and distributed reaction zones regimes (as long as the non-unity burning efficiency, $I_{0}$, is taken into account). A posteriori studies comparing LES of high Karlovitz number flames should be performed in future work.

\section{Acknowledgements}

The authors gratefully acknowledge funding from Air Force Office of Scientific Research (FA9550-12-1-0472 and FA9550-12-1-0144) under supervision of Dr. Chiping Li and Fonds de Recherche du Québec - Nature et Techonologies for financial support. This research used resources of the National Energy Research Scientific Computing Center, a DOE Office of Science User Facility supported by the Office of Science of the U.S. Department of Energy under Contract No. DE-AC02- 
05CH11231. This work used the Extreme Science and Engineering Discovery Environment (XSEDE), which is supported by National Science Foundation grant number ACI-1053575.

\section{References}

[1] H. Pitsch, Large-eddy simulation of turbulent combustion, Ann. Rev. Fluid Mech. 38 (2006) 453 - 482.

[2] J. A. van Oijen, F. A. Lammers, L. P. H. de Goey, Modeling of complex premixed burner systems by using flamelet-generated manifolds, Combust. Flame 127 (3) (2001) $2124-2134$.

[3] O. Gicquel, N. Darabiha, D. Thevenin, Laminar premixed hydrogen/air counterflow flame simulations using flame prolongation of ildm with differential diffusion, Proc. Combust. Inst. 28 (2) (2000) $1901-1908$.

[4] V. Moureau, B. Fiorina, H. Pitsch, A level set formulation for premixed combustion LES considering the turbulent flame structure, Combust. Flame 156 (4) (2009) 801 812.

[5] P. Domingo, L. Vervisch, D. Veynante, Large-eddy simulation of a lifted methane jet flame in a vitiated coflow, Combust. Flame 153 (2) (2008) 415 - 432.

[6] C. Duwig, Study of a filtered flamelet formulation for Large Eddy Simulation of premixed turbulent flames, Flow Turb. Combust. 79 (2007) 433 - 454.

[7] B. Fiorina, R. Vicquelin, P. Auzillon, N. Darabiha, O. Gicquel, D. Veynante, A filtered tabulated chemistry model for LES of premixed combustion, Combust. Flame 157 (2010) $465-475$.

[8] V. Moureau, P. Domingo, L. Vervisch, From large-eddy simulaiton to direct numerical simulation of a lean premixed swirl flame: Filtered laminar flame-pdf modeling, Combust. Flame 158 (2011) 1340 - 1357.

[9] S. Mukhopadhyay, R. Bastiaans, J. van Oijen, L. de Goey, Analysis of a filtered flamelet approach for coarse DNS of premixed turbulent combustion, Fuel 144 (2015) $388-399$.

[10] S. Mukhopadhyay, J. van Oijen, L. de Goey, A comparative study of presumed pdfs for premixed turbulent combustion modeling based on progress variable and its variance, Fuel 144 (2015) $728-740$.

[11] S. Nambully, P. Domingo, V. Moureau, L. Vervisch, A filtered-laminar-flame pdf subgrid scale closure for LES of premixed turbulent flames. part I: Formalism and application to a bluff-body burner with differential diffusion, Combust. Flame 161 (2014) $1756-1774$.

[12] R. Mercier, V. Moureau, D. Veynante, B. Fiorina, LES of turbulent combustion: On the consistency between flame and flow filter scales, Proc. Combust. Inst. 35 (2) (2015) $1359-1366$.

[13] Y. Chen, M. Ihme, Large-eddy simulation of a piloted premixed jet burner, Combust. Flame 160 (2013) $2896-2910$.

[14] C. Duwig, K.-J. Nogenmyr, C.-K. Chan, M. J. Dunn, Large eddy simulations of a piloted lean premixed jet flame using finite-rate chemistry, Combust. Theo. Model. 15 (4) (2011) $537-568$.

[15] B. Savard, B. Bobbitt, G. Blanquart, Structure of a high Karlovitz n-c7h16 premixed turbulent flame, Proc. Combust. Inst. 35 (2) (2015) 1377-1384.

[16] R. Sankaran, E. Hawkes, C. Yoo, J. Chen, Response of flame thickness and propagation speed under intense turbulence in spatially developing lean premixed methane-air jet flames, Combust. Flame 162 (2015) 3294 - 3306.

[17] S. Lapointe, B. Savard, G. Blanquart, Differential diffusion effects, distributed burning, and local extinctions in high Karlovitz premixed flames, Combust. Flame 162 (9) (2015) $3341-3355$.

[18] B. Savard, G. Blanquart, Broken reaction zone and differential diffusion effects in 
a high Karlovitz n-c7h16 premixed turbulent flame, Combust. Flame 162 (5) (2015) 2020 - 2033.

[19] E. Knudsen, H. Kolla, E. Hawkes, H. Pitsch, LES of a premixed jet flame DNS using a strained flamelet model, Combust. Flame 160 (2013) $2911-2927$.

[20] B. Bobbitt, S. Lapointe, G. Blanquart, Vorticity transformation in high Karlovitz number premixed flames, Phys. Fluids 28 (2016) 015101.

[21] A. Aspden, J. Bell, M. Day, S. Woosley, M. Zingale, Turbulenceflame interactions in type ia supernovae, Astrophys. J. 689 (2008) $1173-1185$.

[22] A. Aspden, J. Bell, S. Woosley, Distributed flames in type ia supernovae, Astrophys. J. $710(2010) 1654-1663$.

[23] A. Aspden, M. Day, J. Bell, Turbulence-flame interactions in lean premixed hydrogen: transition to the distributed burning regime, J. Fluid Mech. 680 (2011) $287-320$.

[24] A. Aspden, M. Day, J. Bell, Turbulence-chemistry interaction in lean premixed hydrogen combustion, Proc. Combust. Inst. 35 (2015) 1321-1329.

[25] A. Aspden, M. Day, J. Bell, Lewis number effects in distributed flames, Proc. Combust. Inst. 33 (2011) $1473-1480$.

[26] A. Poludnenko, E. Oran, The interaction of high-speed turbulence with flames: Global properties and internal flame structure, Combust. Flame 157 (2010) 995 - 1011.

[27] A. Poludnenko, E. Oran, The interaction of high-speed turbulence with flames: Turbulent flame speed, Combust. Flame 158 (2011) $301-326$.

[28] A. Poludnenko, Pulsating instability and self-acceleration of fast turbulent flames, Phys. Fluids 27 (014106) (2015) $1-25$.

[29] C. Rosales, C. Meneveau, Linear forcing in numerical simulations of isotropic turbulence: Physical space implementations and convergence properties, Phys. Fluids 17 (2005) $0951061-8$.

[30] P. Carroll, G. Blanquart, The effect of velocity field forcing techniques on the nature and evolution of the Karman-Howarth equation, Journal of Turbulence 15 (2014) 429 -448 .

[31] T. Lundgren, Linearly forced isotropic turbulence, in: Annual Research Briefs (Center for Turbulence Research), Stanford, 2003, 2003, pp. 461-473.

[32] N. Burali, S. Lapointe, B. Bobbitt, Y. Xuan, G. Blanquart, Assessment of the constant non-unity lewis number assumption in chemically-reacting flows, Combust. Theor. Model. (2016) accepted.

[33] B. Savard, Characterization and modeling of premixed turbulent $n$-heptane flames in the thin reaction zone regime, Ph.D. thesis, California Institute of Technology (2015).

[34] M. Ihme, H. Pitsch, Prediction of extinction and reignition in nonpremixed turbulent flames using a flamelet/progress variable model. 1. A priori study and presumed PDF closure, Combust. Flame 155 (2008) 70 - 89.

[35] M. Ihme, H. Pitsch, Prediction of extinction and reignition in nonpremixed turbulent flames using a flamelet/progress variable model. 2. A posteriori study with application to sandia flames D and E, Combust. Flame 155 (2008) 90 - 107.

[36] S. Menon, P. Boettcher, G. Blanquart, Enthalpy based approach to capture heat transfer effects in premixed combustion, Combust. Flame 160 (2012) 1242-1253.

[37] L. Vervisch, P. Domingo, G. Lodato, D. Veynante, Scalar energy fluctuations in largeeddy simulation of turbulent flames: Statistical budgets and mesh quality criterion, Combust. Flame 157 (2010) $778-789$.

[38] E. Knudsen, S. Kim, H. Pitsch, An analysis of premixed flamelet models for large eddy simulation of turbulent combustion, Phys. Fluids 22 (2010) $1151091-24$.

[39] S. Lapointe, G. Blanquart, Fuel and chemistry effects in high Karlovitz premixed flames, Combust. Flamedoi:10.1016/j.combustflame.2016.01.035.

[40] D. Bradley, How fast can we burn?, Proc. Combust. Inst. 24 (1992) 247 - 262.

[41] B. Savard, G. Blanquart, An a priori model for the effective species lewis numbers in premixed turbulent flames, Combust. Flame 161 (2014) 1547 - 1557.

[42] E. Knudsen, E. Richardson, E. Doran, H. Pitsch, J. Chen, Modeling scalar dissipation and scalar variance in large eddy simulation: Algebraic and transport equation closures, Phys. Fluids 24 (5) (2012) $0551031-24$.

[43] A. Moureau, O. Teytaud, J.-P. Bertoglio, Optimal estimation for large-eddy simulation of turbulence and application to the analysis of subgrid models, Phys. Fluids 18 (10) (2006) 105101 1-10.

[44] G. Balarac, H. Pitsch, V. Raman, Development of a dynamic model for the subfilter 
scalar variance using the concept of optimal estimators, Physics of Fluids 20 (3). doi:http://dx.doi.org/10.1063/1.2896287.

URL http://scitation.aip.org/content/aip/journal/pof2/ $20 / 3 / 10.1063 / 1.2896287$

[45] B.Fiorina, O. Gicquei, L. Vervisch, S. Carpentier, N. Darabiha, Premixed turbulent combustion modeling using tabulated detailed chemistry and pdf, Proc. Combust. Inst. 30 (2005) $867-874$.

[46] F. Hernandez-Perez, F. Yuen, C. Groth, O. Gulder, LES of a laboratory-scale turbulent premixed bunsen flame using FSD, PCM-FPI and thickened flame models, Proc. Combust. Inst. 33 (2011) $1365-1371$.

\section{A Appendix: Presumed PDF}

A common approach in tabulated chemistry combustion models is to presume the shape of the probability density function of the progress variable. A $\beta$-function PDF is often assumed $[45,46]$. The $\beta$-probability density function is defined as:

$$
P\left(c \mid \tilde{c}, c_{v}\right)=\frac{c^{a-1}(1-c)^{b-1}}{\int_{0}^{1} c^{a-1}(1-c)^{b-1} \mathrm{~d} c},
$$

where $a$ and $b$ are determined from $\tilde{c}$ and its variance:

$$
a=\tilde{c}\left(\frac{\tilde{c}(1-\tilde{c})}{c_{v}}-1\right), \quad b=a\left(\frac{1}{\tilde{c}}-1\right)
$$

for $c$ normalized between 0 and 1 .

Another approach consists of using spatially filtered laminar flames to generate the lookup table [11]. A reference one-dimensional flame is filtered at different filter widths and the filtered fields are then tabulated against the filtered progress variable $\tilde{c}$ and its variance. Moureau et al. [8] showed that the use of a variable filter size led to better predictions than using the LES grid size as the filter width. This approach is equivalent to convoluting the laminar progress variable source term with a PDF obtained from filtered laminar flames, labeled FLF-PDF. The sub-filter PDF of the FLF model is

$$
P\left(c \mid \tilde{c}, S_{c}\right)=\frac{\rho(c) F_{\Delta}\left(\tilde{x}^{\Delta}(\tilde{c}, \Delta)-x(c)\right)}{\bar{\rho}\left(\tilde{c}, S_{c}\right)|\nabla c|},
$$

where $\Delta=\Delta\left(\tilde{c}, S_{c}\right)$ is the filter size that needs to be applied to the laminar flame to match the sub-grid scale scalar variance, and $S_{c}=c_{v} /[\tilde{c}(1-\tilde{c})]$ is the unmixedness factor.

\section{B Appendix: Effect of integral length scale}

Case $\mathrm{B}^{4}$ was performed at the same Karlovitz number as case B but with a larger integral length scale. This makes possible the use of larger filter widths and allows to study the 
effects of integral length scale (or Reynolds number) on the chemical source terms. This is investigated by comparing the conditional means and probability density functions of $\dot{\omega}_{c}$ for both cases. The source terms are almost identical between cases $\mathrm{B}$ and $\mathrm{B}^{4}$ with similar conditional means and probability density functions. This indicates that there are no effects of integral length scale on the chemical source terms despite the low Reynolds number of cases B.

Furthermore, it was shown in recent work [20] that vorticity characteristics are only affected by the small turbulent scales. The present conclusions on LES with tabulated chemistry using presumed PDFs are thus expected to hold at higher Reynolds numbers.

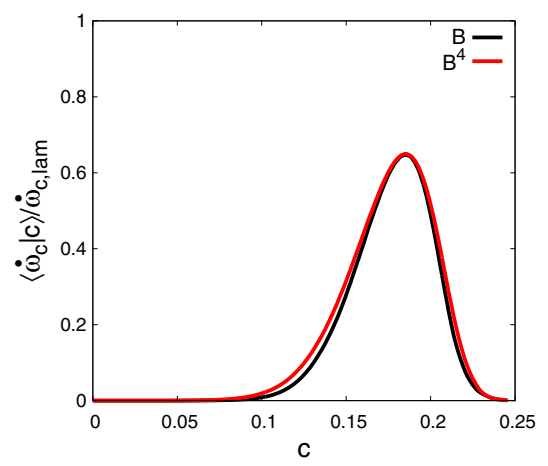

(a) Conditional mean

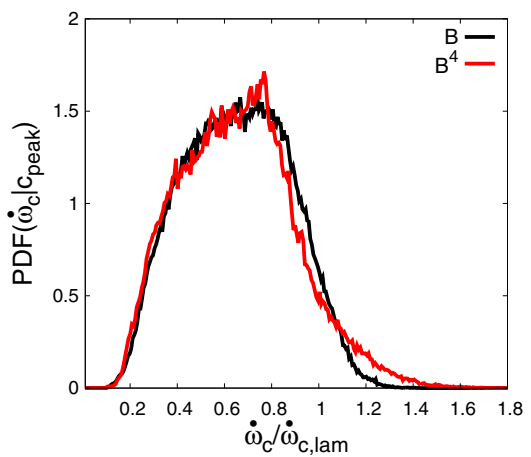

(b) PDF of $\dot{\omega}_{c}$

Fig. B.1. Conditional means (a) and probability density functions (b) of the progress variable source term from detailed chemistry, non-unity Lewis number DNS for case B (black lines) compared to case $\mathrm{B}^{4}$ (red lines). 